\title{
Avaliação de resíduos de mineração de rochas cristalinas para uso como agregado em concreto segundo sua petrografia, índices físicos e resistência mecânica
}

\section{Evaluation of crystalline rock mining waste for use as a concrete aggregate according to its petrography, physical indexes and mechanical strength}

\author{
Ana Beatriz Azevedo de Medeiros ${ }^{1}$, Maria del Pilar Durante Ingunza ${ }^{1}$, \\ Antônio Carlos Galindo ${ }^{2}$, Rubens Maribondo do Nascimento ${ }^{3}$
}

\footnotetext{
${ }^{1}$ Universidade Federal do Rio Grande do Norte - Campus Universitário, Programa de Pós Graduação em Engenharia Civil, Rua das Engenharias, s/n, CEP: 59078-970, Lagoa Nova, Natal, RN, Brasil

${ }^{2}$ Universidade Federal do Rio Grande do Norte - Campus Universitário, Programa de Pós Graduação em Geodinâmica e Geofísica, Rua das Engenharias, s/n, CEP: 59078-970, Lagoa Nova, Natal , RN, Brasil.

${ }^{3}$ Universidade Federal do Rio Grande do Norte - Campus Universitário, Programa de Pós Graduação em Ciência e Engenharia de Materiais, Rua das Engenharias, s/n, CEP: 59078-970, Lagoa Nova, Natal, RN, Brasil.

e-mail: aninhabia@ufrn.edu.br, durante@ct.ufrn.br, galindo@geologia.ufrn.br, rmaribondo@ufrnet.br
}

\section{RESUMO}

$\mathrm{Na}$ atualidade, os resíduos de mineração vem sendo considerados como matéria prima nos programas de sustentabilidade ambiental, com o objetivo de minimizar os impactos decorrentes da atividade mineradora. Diante disso, o presente trabalho propõe analisar os resíduos de mineração de pegmatito para uso em agregado para concretos. As amostras foram caracterizadas petrograficamente, segundo seu potencial reativo à Reação Álcali-Agregado sob a norma ABNT 15577-3 e foram realizados ensaios para avaliação dos índices físicos e da resistência mecânica. Verificou-se entre as amostras de pegmatitos que duas delas obtiveram inocuidade para a reação álcali-agregado, por conter teores de fases deletéreas à RAA abaixo do limite aceitável pela norma. Entretanto, as demais amostras apresentaram potencial reatividade à RAA, por conter ora quartzo deformado, ora ambas as fases deletéras identificadas de acordo com a norma utilizada: quartzo deformado e microgranular. Os valores de resistência mecânica assumiram valores abaixo dos valores médios apresentados pela literatura consultada. Esse fato se justifica pelo grau de alteração observado nas amostras estudadas. Comprovou-se que os resíduos de lavra foram submetidos às ações intempéricas, tendo como resultado uma queda nos valores de resistência mecânica esperados. Considerando os índices físicos, as amostras apresentaram bons resultados no que diz respeito à capacidade de absorção de umidade.

Palavras-chave: Resíduos de mineração- agregado em concreto- petrografia- índices físicos- resistência mecânica

\section{ABSTRACT}

Currently, mining waste has been take an important role as a raw material in environmental sustainability programs, with the objective of minimizing the impacts resulting from mining activity. Given this, this research proposes to analyze pegmatite mining waste for use in aggregate for concrete. The samples were petrographically characterized according to their reactive potential to the Alkali-Aggregate Reaction under standard ABNT 15577-3 and tests were performed to evaluating the physical indexes and mechanical strength. It was found among the dimensions of pegmatites that two of them shows innocuous for alkaliaggregate reaction, because it contains levels of deleterious phases for RAA below the limit acceptable by the norm. Though, the other samples showed potential reactivity to RAA, because either contains deformed quartz, or both deleterious phases identified according to the standard used: deformed quartz and microgranular. The mechanical resistance assumed values below the average values presented by the consulted literature. 
This fact is justified by the degree of alteration observed in the studied samples. Mining residues were found to have been subjected to weathering, resulting in a drop in expected mechanical strength values. Considering the physical indexes, the samples showed good results regarding the moisture absorption capacity.

Keywords: Mining waste-concrete aggregate-petrography-physical indexes- mechanical resistance

\section{INTRODUÇÃO}

De acordo com IBRAM [1], no ano de 2018 o volume de bens minerais exportados no Brasil atingiu 409 milhões de toneladas, representando em dólares 29,9 bilhões. Sendo assim, a quantidade de resíduos gerados no processo de obtenção dos minérios exportados é bem considerável e oferece uma boa fonte de resíduos para o setor da construção civil.

Durante a última década, a necessidade de encontrar soluções eficientes e sustentáveis só cresceu. A reutilização/reciclagem desses materiais tem a capacidade de reduzir o volume de resíduos no meio ambiente. Tal fato contribui diretamente para redução dos custos de gerenciamento, restauração e à prevenção de problemas relacionados à poluição MURLIDHAR et al. [2].

BARBOSA et al. [3] utilizaram resíduo de granito a fim de empregar como material cimentício, obtendo bons resultados segundo à resistência à compressão com o aumento do tempo de moagem desses resíduos. Já PAVEZ et al. [4] utilizou escória de cobre em argamassa como substituto parcial de cimento.

Como aplicação de agregados, SALGUERO et al. [5], GONÇALVES E MOURA [6], SANTOS et al [7], MOREIRA et al. [8], FRANCO et al. [9], SANTOS et al. [10], PASSUELO et al [11] propõem substituição parcial a total agregado miúdo convencional por resíduos de manganês, serragem de granito, britagem de granito, quartzito, ferro, carvão e ágata respectivamente.

Considerando os agregados graúdos, diferentes tipos de rochas obtiveram bons resultados para substituição: mármore [12-14], arenito [15], granito [14] e FILITO [16].

Em trabalho recente, BRAGAGNOLO \& KORF [17] apontaram as técnicas analíticas, entre elas a microscopia óptica, como de fundamental importância para a correta aplicação de resíduos no concreto. Da mesma forma, no âmbito internacional, HEMMATI et al [18] destacam o papel da mineralogia no estudo do comportamento mecânico de rochas cristalinas.

O estado do Rio Grande do Norte possui notável tradição no setor mineiro. Esse estado, no último levantamento realizado pelo Departamento Nacional de Produção Mineral (DNPM) [19] comercializou em torno de 455 milhões de reais em bens minerais, dentre os quais ferro, tungstênio, tântalo e outros bens minerais como calcário, rochas britadas, rochas ornamentais e argila.

Sendo o Rio Grande do Norte um estado com forte demanda de resíduos minerários, o objetivo desse trabalho é caracterizar resíduos de lavra de pegmatitos segundo seu potencial à reação álcali-agregado a nível petrográfico, índices físicos e resistência mecânica para fins de aproveitamento como agregado graúdo.Utilizar preferencialmente como estrutura geral a seguinte sequência:

\section{MATERIAIS E MÉTODOS}

\subsection{Materiais}

Para fins de desenvolvimento da pesquisa, foram utilizados resíduos de lavra de pegmatito numa mina alocada no município de Parelhas, estado do Rio Grande do Norte. As amostras de pegmatito foram coletadas no município de Parelhas, conforme o mapa abaixo (figura 1): 


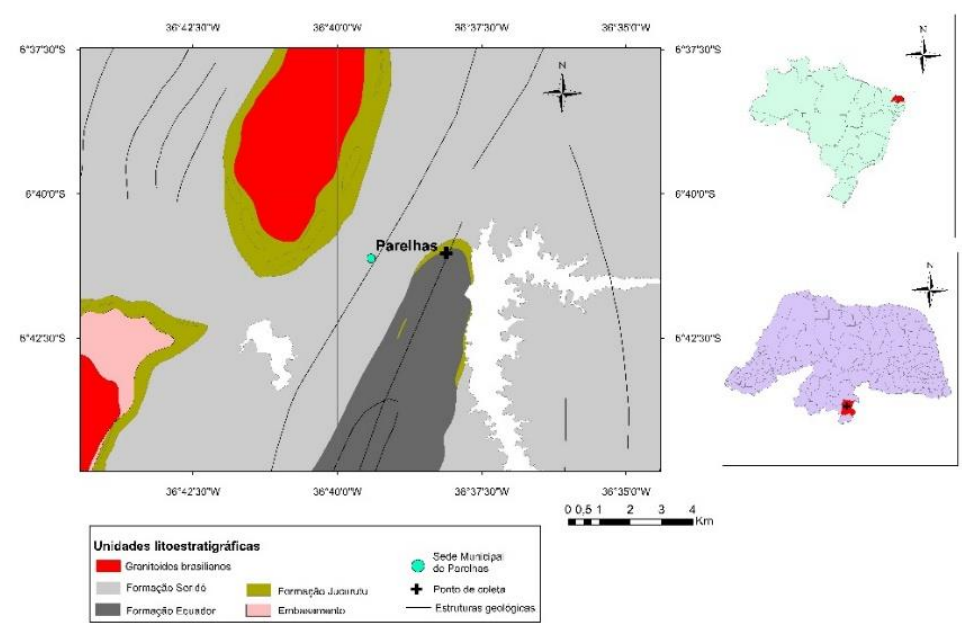

Figura 1: Mapa de localização do ponto de coleta dos pegmatitos. Fonte:CPRM [20]

\subsection{Métodos}

\subsection{Amostragem}

A amostragem do pegmatito foi feita de acordo com a norma ABNT NBR 26 [21] e resultou na coleta de 9 amostras representativas do pegmatito analisado.

\subsection{Análise petrográfica}

A análise petrográfica foi realizada no laboratório de microscopia óptica no departamento de geologia da UFRN. A norma utilizada para avaliar o potencial à reatividade das amostras foi a norma ABNT 15777 parte 3 [22]. Os passos para análise das amostras estão descritos no esquema abaixo (figura 2):

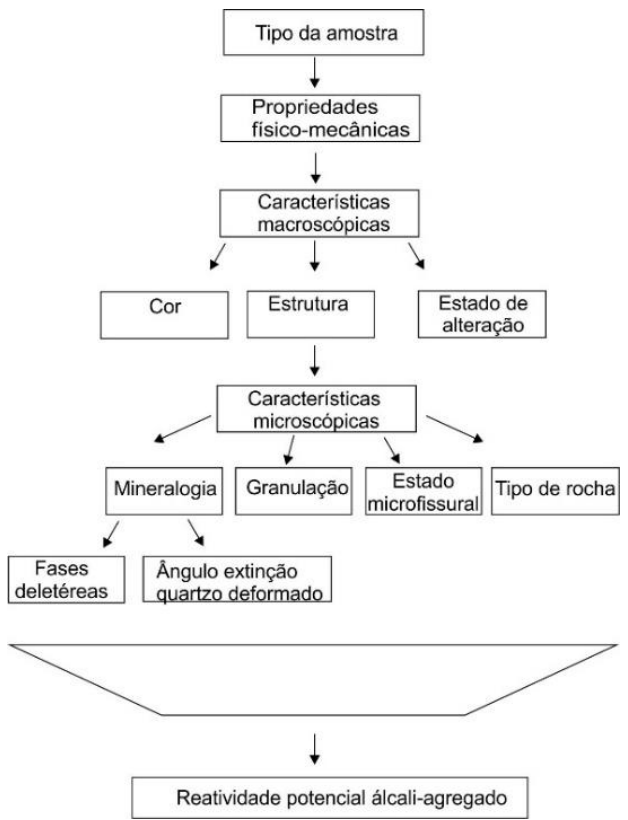

Figura 2: Esquema dos passos metodológicos para análise petrográfica das amostras de acordo com a norma ABNT 15577-3 [22].

\section{4 Índices físicos}

Os índices físicos foram determinados segundo duas normas distintas: a norma ABNT NBR NM 53: 2003 [23] foi utilizada para determinar a massa específica e absorção. Já para determinação da porosidade foi utili- 
zada a norma ABNT NBR 7418:1987 [24].

\subsection{Resistência mecânica}

A resistência mecânica foi determinada indiretamente através do ensaio esclerométrico. Tanto os índices físicos quanto a resistência mecânica foram realizados no laboratório de construção civil, no departamento de engenharia civil da UFRN.

\section{RESULTADOS}

\subsection{Análise petrográfica}

As fotomicrografias de cada amostra estudada estão apresentadas na figura 3 e segue a ordem apresentada na tabela 1, abaixo:

Tabela 1: Relação da amostra e sua respectiva fotomicrografia

\begin{tabular}{l|l}
\hline AMOSTRA & FOTOMICROGRAFIA \\
\hline AMPA-01 & A \\
\hline AMPA-02 & B \\
\hline AMPA-03 & C \\
\hline AMPA-04 & D \\
\hline AMPA-05 & E \\
\hline AMPA-06 & F \\
\hline AMPA-07 & G \\
\hline AMPA-08 & H \\
\hline AMPA-09 & $\mathrm{I}$ \\
\hline
\end{tabular}
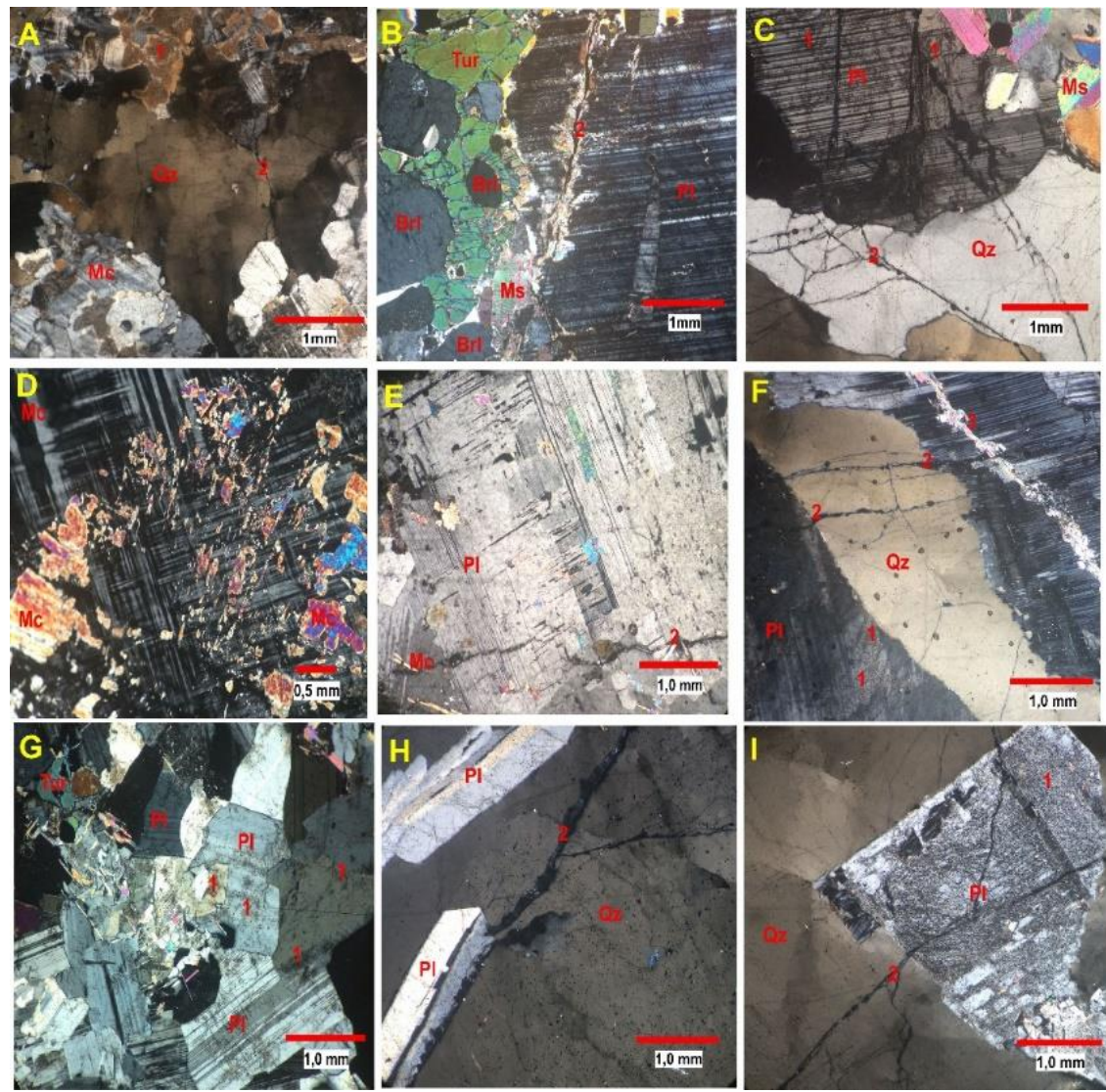

Figura 3: Fotomicrografias das 9 amostras de pegmatito analisadas. A fotomicrografia A corresponde à amostra AMPA01 e assim sucessivamente para as demais amostras. Genericamente, percebe-se pontos de alteração nas amostras (1) e microfissuramento (2). Nas amostras A,C,F,H,I o Quartzo (Qz) apresenta deformação, caracterizado pela extinção ondu- 
lante, um dos aspectos requeridos para diagnóstico ao potencial à reação álcali-agregado. Além do Quartzo (Qz), outras fases mineralógicas foram identificadas como a Microclina (Mc) (fotomicrografias A, D, E), o Plagioclásio (Pl) (fotomicrografias B,C,E,F,G,H,I), Turmalina (Tur) (fotomicrografias B, G), Berilo (Brl) (fotomicrografia B), Muscovita (Ms) (fotomicrografias B, C).

\section{2 Índices físicos}

Os valores de absorção, porosidade e densidade real estão ilustradas nos gráficos abaixo, nas figuras 4, 5 e 6

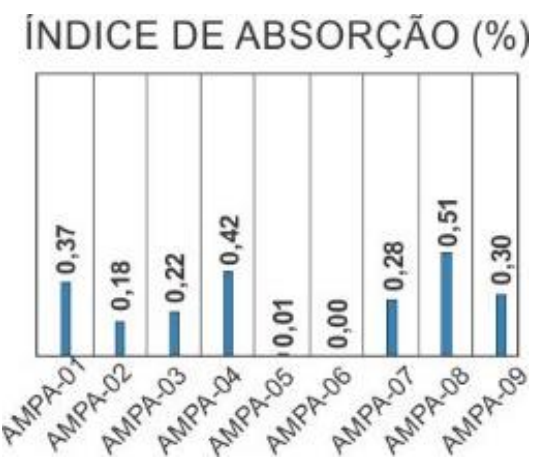

Figura 4:Valores de absorção (em \%) para cada amostra analisada.

POROSIDADE (\%)

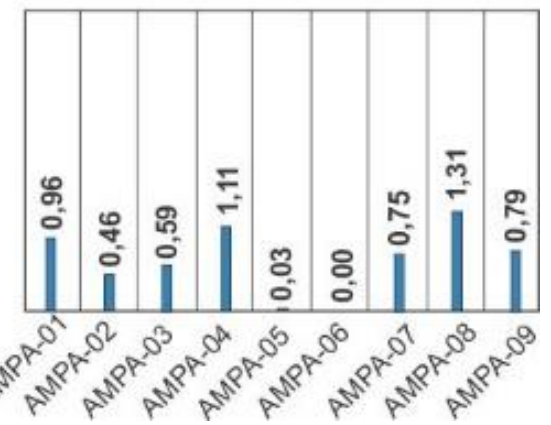

Figura 5: Valores de porosidade (em \%) para cada amostra

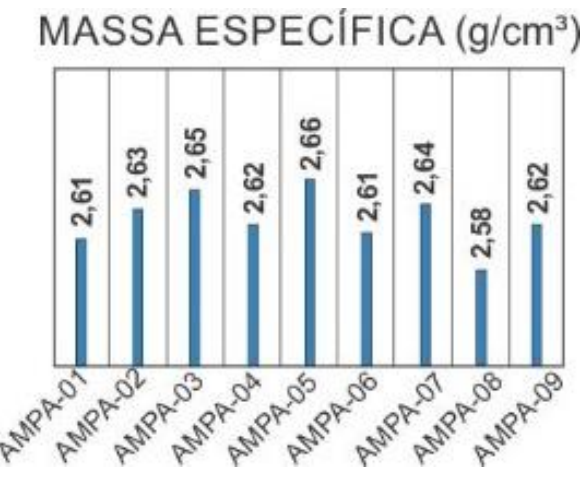

Figura 6: Valores de massa específica para cada amostra $\left(\mathrm{g} / \mathrm{cm}^{3}\right)$

\subsection{Resistência mecânica}

Na Figura 7, abaixo, estão apresentados os valores de resistência à compressão uniaxial, obtidos no ensaio com esclerômetro de Schmidt: 


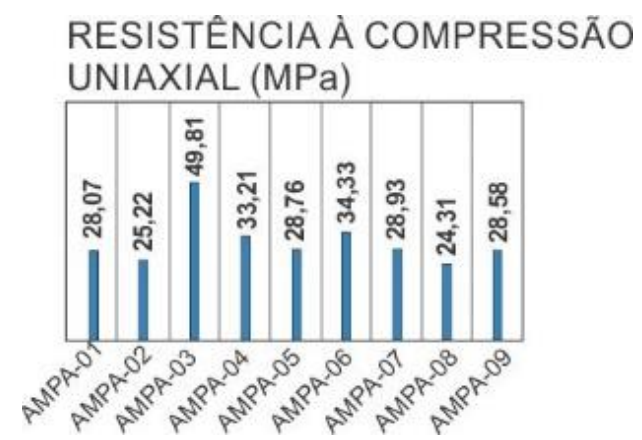

Figura 7: Valores de resistência mecânica (Mpa) para cada amostra.

\section{DISCUSSÃO}

\subsection{Análise petrográfica}

De acordo com os aspectos a serem descritos pela norma ABNT 15777-3[22], as amostras AMPA-04 e AMPA-05 foram classificadas como potencialmente inócua à RAA. Já as demais amostras (AMPA-01, AMPA02, AMPA,03, AMPA-06, AMPA-07, AMPA-08 e AMPA-09) foram classificadas como potencialmente reativa à RAA.

Das 9 amostras classificadas com potencial à reatividade, as fases deletéreas identificadas foram o quartzo deformardo (com ângulo de extinção ondulante variando de $14^{\circ}$ a $26^{\circ}$ ) e o quartzo microgranular. De todas as fases deletéreas atribuídas pela norma, duas estão presentes nas amostras, de acordo com as tabelas 2 e 3 :

Tabela 2: Diagnóstico de presença de quartzo deformado por amostra.

\begin{tabular}{l|l}
\hline QUARTZO DEFORMADO & AMOSTRA \\
\hline $\operatorname{sim}$ & AMPA-01 \\
\hline $\operatorname{sim}$ & AMPA-02 \\
\hline $\operatorname{sim}$ & AMPA-03 \\
\hline $\operatorname{sim}$ & AMPA-06 \\
\hline $\operatorname{sim}$ & AMPA-07 \\
\hline $\operatorname{sim}$ & AMPA-08 \\
\hline $\operatorname{sim}$ & AMPA-09 \\
\hline 7 & Quartzo deformado \\
\hline
\end{tabular}

Tabela 3: Diagnóstico da presença de quartzo microgranular.

\begin{tabular}{l|l}
\hline $\begin{array}{l}\text { QUARTZO MICROGRANU- } \\
\text { LAR }\end{array}$ & AMOSTRA \\
\hline sim & AMPA-01 \\
\hline não & AMPA-02 \\
\hline não & AMPA-03 \\
\hline não & AMPA-06 \\
\hline não & AMPA-07 \\
\hline não & AMPA-08 \\
\hline não & AMPA-09 \\
\hline 1 & Quartzo microgranular \\
\hline
\end{tabular}

Das amostras classificadas como potencialmente reativas, a amostra AMPA-01 apresentou as duas fases deletéreas identificadas na análise petrográfica, de acordo com a tabela 4, abaixo: 
Tabela 4: Diagnóstico de ambas as fases deletéreas nas amostras.

\begin{tabular}{l|l}
\hline AMBAS & AMOSTRA \\
\hline sim & AMPA-01 \\
\hline não & AMPA-02 \\
\hline não & AMPA-03 \\
\hline não & AMPA-06 \\
\hline não & AMPA-07 \\
\hline não & AMPA-08 \\
\hline não & AMPA-09 \\
\hline 1 & Ambos \\
\hline
\end{tabular}

Com base nos resultados das análises petrográficas, todas as amostras diagnosticadas como potencialmente reativas à Reação Álcali-Agregado (RAA) contém teor de quartzo deformado acima de 5\%, caracterizando-as como reativas, segundo a norma 15577-3[22]. Porém, ressalva-se: as amostras que apresenta apenas o quartzo deformado como fase deletérea são passíveis de serem reconsideradas para um diagnóstico global, considerando os trabalhos publicados. WIGUN [25], concluiu que as fases mais reativas foram o quartzo microgranular e o quartzo com desenvolvimento de subgrão (é uma forma precedente do quartzo microgranular, conforme avança sua deformação). Por sua vez, ANDRADE et al [26] utilizaram testes de expansão para analisar as fases: quartzo com extinção ondulante (quartzo deformado) moderada a forte, quartzo microcristalino e feldspatos alcalino, obtendo resultados de inocuidade para amostras que apresentaram apenas extinção ondulante (quartzo deformado). Já PRADO [27], através de programa experimental, concluiu que as amostras contendo apenas quartzo com extinção ondulante (quartzo deformado) são muito menos susceptíveis a desenvolver RAA, por possuir menos defeitos cristalográficos (em relação às demais fases deletéreas que sofreram mais deformação) e portanto é menos reativo. E ainda, GRATTAN-BELLEW [28] reforça que a extinção ondulante no quartzo é a manifestação preliminar da deformação, e portanto, da reatividade à RAA, evoluindo para o grau de quartzo microcristalino e maior reatividade. Portanto, é importante considerar estudos complementares com essas amostras.

\section{2 Índices físicos}

As tabelas a seguir (tabelas 5, 6 e 7) apresentam valores de densidade, absorção e porosidade, respectivamente, com base na literatura:

Tabela 5: Valores de absorção de algumas litologias.

\begin{tabular}{l|l}
\hline TIPO DE ROCHA & ABSORÇÃO $(\%)$ \\
\hline Granito pegmatitítico & 0,96 \\
\hline Basalto maciço & 0,21 \\
\hline Diorito & 0,42 \\
\hline Anfibolito & 0,19 \\
\hline Biotita gnaisse & 0,85 \\
\hline Xisto & 1,49 \\
\hline
\end{tabular}

Fonte: Ahmed et al [14] 
Tabela 6: Valores de porosidade de alguns tipos de rochas.

\begin{tabular}{l|l}
\hline TIPO DE ROCHA & POROSIDADE (\%) \\
\hline Dolerito & 1,90 \\
\hline Basalto & 1,35 \\
\hline Biotita gnaisse & 0,54 \\
\hline Anfibolito & 2,19 \\
\hline Pegmatito & 0,51 \\
\hline Quartzito & 0,48 \\
\hline Gnaisse porfirítico & 1,28
\end{tabular}

Tabela 7: Valores de densidade para alguns tipos de rochas.

\begin{tabular}{l|l}
\hline TIPO DE ROCHA & DENSIDADE $\left(\mathrm{G} / \mathrm{CM}^{3}\right)$ \\
\hline Dolerito & 3,012 \\
\hline Basalto & 3,001 \\
\hline Biotita gnaisse & 2,636 \\
\hline Anfibolito & 2,859 \\
\hline Pegmatito & 2,701 \\
\hline Quartzito & 2,617 \\
\hline Gnaisse porfirítico & 2,718 \\
\hline \multicolumn{1}{c}{ Fonte: Singh et al [29] }
\end{tabular}

Os resultados dos ensaios referentes aos índices físicos (absorção, porosidade e densidade), apresentados nos gráficos (figuras 4, 5 e 6) estão de acordo com os valores apresentados nas tabelas 5, 6 e 7. Na tabela 5 , o valor médio de absorção é de $0,96 \%$., enquanto que as amostras estudadas nesse trabalho tiveram valores variando de 0 a $0,51 \%$ (Figura 4). Em relação à porosidade, o valor médio de porosidade é de 0,51\% (Tabela 6). Os valores de porosidade obtidos com os ensaios descritos nos métodos desse trabalho, variaram de 0 a $1,31 \%$ (Figura 5). Quanto à densidade, os valores obtidos neste trabalho variaram de 2,58g/cm a $2,66 \mathrm{~g} / \mathrm{cm}^{3}$ (Figura 6) e se enquadram dentro dos valores de densidade dados pela literatura (Tabela 7). Os resultados dos ensaios de absorção e porosidade permitem concluir que, por ter valores muito baixos, no uso como agregado para concreto a possibilidade de desenvolvimento de RAA será menor, sendo a umidade um dos fatores que aceleram a reação que dá origem a essa patologia.

\subsection{Resistência mecânica}

A tabela 8 a seguir ilustra valores de resistência mecânica de algumas tipologias de rochas, de acordo com a literatura:

Tabela 8: Valores médios de resistência à compressão para diferentes tipos de rochas.

\begin{tabular}{l|l}
\hline ROCHA & VALORES DO TESTE DE SCHMIDT (MPA) \\
\hline Muscovita xisto & $46 \pm 13,8$ \\
\hline Pegmatito & 82,5 \\
\hline Biotita xisto & $75 \pm 24,8$ \\
\hline Biotita gnaisse & $81,1 \pm 21,0$ \\
\hline
\end{tabular}

Fonte: Sjöberg,([30]

Comparando com o valor médio de resistência mecânica do pegmatito (82,5Mpa) da literatura (Tabela 8$)$, 
nota-se que os valores obtidos no programa experimental desse trabalho foram mais baixos. No presente trabalho, os valores variaram de 24,31 Mpa a 49,81 Mpa (Figura 7). Porém, destaca-se que as amostras utilizadas para esse estudo foram submetidas a ações intempéricas, confirmada nas análises petrográficas sob alterações minerais e fraturas. Tais intempéries são responsáveis por alterar o grau de coesão na rocha, interferindo na própria resistência mecânica.

\section{CONCLUSÕES}

Essa pesquisa buscou avaliar resíduos de lavra de pegmatito segundo sua potencialidade à reação álcali agregado, índices físicos e resistência mecânica através de ensaio esclerométrico.

Das 9 amostras analisadas, duas amostras apresentaram potencial inocuidade à reação álcali agregado. Uma delas apresenta as duas fases deletéreas presentes: quartzo deformado e microgranular (AMPA-01).

Segundo os índices físicos (densidade real, absorção e porosidade), os valores médios assumidos por cada amostra condizem com os valores da literatura.

Os valores de resistência mecânica assumiram valores abaixo dos valores médios apresentados pela literatura consultada. Este fato se justifica pelo grau de alteração observado nas amostras estudadas. Os resíduos de lavra estavam submetido às ações intempéricas, tendo como resultado uma queda nos valores de resistência mecânica esperados.

\section{AGRADECIMENTOS}

Agradecemos ao Programa de Pós Graduação em Engenharia Civil pela oportunidade de realizar essa pesquisa e às empresas as quais forneceram as amostras analisadas.

\section{BIBLIOGRAFIA}

[1] IBRAM. Instituto Brasileiro de Mineração. Relatório Anual de Atividades julho 2018 a junho 2019. Disponível em: http://www.portaldamineracao.com.br (acesso em 31 de agosto,2019).

[2] MURLIDHAR, B.R., MOHAMAD, E.T., ARMAGHANI, D.J., et al. Potential alkali silica reactivity of various rock types in an aggregate granite quarry. Measurement. pp. 221-231, 2016.

[3] BARBOSA, J. M., CARVALHO, J.M.F., CARMO E SILVA, K.D. et al. Influence of grinding on the pozzolanic activity of granite residue. REM, Int. Eng. J., Ouro Preto, n. 72, v.3, pp. 395-404, jul. sep. 2019.

[4] PAVEZ, O., NAZER, A., RIVERA, et al. Copper slag from different dumps in the Atacama Region used in mortars as partial replacement of cement. Revista Matéria. v.24, n. 1, 2019.

[5] SALGUERO, F., GRANDE, J.A., VALENTE,T., et al. A. Recycling of manganese gangue materials from waste-dumps.in the Iberian Pyrite Belt - Application as filler for concrete production. Construction and Building Materials. 2014

[6] GONÇALVES, J.P. MOURA,W.A. Reciclagem do resíduo do beneficiamento de rochas ornamentais na construção civil. III SRONE - Recife-PE-Brasil, 2002.

[7] SANTOS, R.A., LIRA, B.B., RIBEIRO, A.C.M., et al., Argamassa Com Substituição De Agregado Natural Por Resíduo De Britagem De Granito. HOLOS, Ano 28, v. 5. 2012.

[8] MOREIRA, C., MEDINA, A.P., LOURENÇO, L., et al,. Estudo da substituição do agregado muído natural pelo resíduo da pedra de pirenópolis em concreto de cimento portland. R. Eng. Constr. Civ., Curitiba PR, v.1, n.2, pp. 12-23, jul./dez., 2014.

[9] FRANCO, L.C., SANTOS, D.H., ROSA, P.P.G. et al., Aplicação de Rejeito de Mineração como Agregado Para a Produção De Concreto. Anais Do $56^{\circ}$ Congresso Brasileiro Do Concreto - CBC2014 - 56CBC. 2014.

[10] SANTOS, C.R., TUBINO, R.M.C., SCHNEIDER, I.A.H., et al., Processamento mineral e caracterização de rejeito de carvão mineral para produção de blocos de concreto para pavimentação. Revista IBRACON de estruturas e materiais. v. 8, n. 1. 2015.

[11] PASSUELLO, A.C., VENQUIARUTO. S., ABREU, A.G., et al., Valorização de resíduos de ágata em argamassas e concretos: avaliação do ciclo de vida. Revista Matéria. v.24, n.2, 2019.

[12] KORE, S.D., VYAS, A.K. Impact of marble waste as coarse aggregate on properties of lean cement concrete. Case studies in construction materials, V. 4, pp. 85-92, 2016. 
[13] DATTATRAYA, K.S., VYAS, A.K. Performance Evaluation of Concrete using Marble Mining Waste. JOURNAL OF CIVIL ENGINEERING, v. 11, Issue 2, 2016.

[14] AHMED, A.A.M., KAREEM, K.H.A., ALTOHAMY, A.M. et al., An Experimental Study On The Availability Of Solid Waste Of Mines And Quarries As Coarse Aggregate In Concrete Mixes. Journal of Engineering Sciences Assiut University Faculty of Engineering, v. 42, n. 3. Pp. 876-890, May 2014.

[15] KUMAR, S., GUPTA, R.C., SHRIVASTAVA, S. et al., Preliminary study on the use of quartz sandstone as a partial replacement of coarse aggregate in concrete based on clay content, morphology and compressive strength of combined gradation. Construction and Building Materials. pp. 103-108, 2016.

[16] ADOM-ASAMOAH, M., AFRIFA, R.O. Investigation on the flexural behaviour of reinforced concrete beams using phyllite aggregates from mining waste. Materials and Design n. 32, pp. 5132-5140, 2011.

[17] BRAGAGNOLO, L., KORF, E.P. Aplicação de resíduos na fabricação de concreto: como técnicas analíticas de caracterização podem auxiliar na escolha preliminar do material mais adequado?. Matéria [online]. v.25, n. 1, 2020

[18] HEMMATI, A., GHAFOORI, M., MOOMIVAND, H., et al., The effect of mineralogy and textural characteristics on the strength of crystalline igneous rocks using image-based textural quantification. Engineering Geology, v. 266. 2020.

[19] DNPM, Anuário Mineral Brasileiro, Brasília-DF, 2010, disponível em: <http://www.dnpm.gov.br/relatorios/amb/Completo_2010.pdf>, acesso em: 01 de novembro de 2017.

[20] CPRM. Companhia de Pesquisa de Recursos Minerais. Mapa estadual do Rio Grande do Norte 1:1.000.000. Acesso: agosto de 2007.

[21] ABNT NBR NM 26:2000 agregados-amostragem. Rio de Janeiro,2001.

[22] ABNT. NBR 15577-3: agregados: reatividade álcali-agregado: parte 3: análise petrográfica para verificação da potencialidade reativa de agregados em presença de álcalis do concreto. Rio de Janeiro, 2008.

[23] ABNT NBR NM:53. Agregado graúdo - Determinação de massa específica, massa específica aparente e absorção de água.

[24] ABNT NBR 7418:1987. Lastro-padrão - Determinação da massa específica aparente, da absorção de água e da porosidade aparente do material.

[25] WIGUM, B.J, Examination of microstructural features of Norwegian cataclastic rocks and their use for predicting alkali-reactivity in concrete, Engineering Geology (40), pp. 195-214, 1995.

[26] ANDRADE,T. "Histórico de casos de RAA ocorridos recentemente em fundações de edifícios na região metropolitana de Recife". In: Simpósio sobre reação Álcali-Agregado em estruturas de concreto, 2. 2006. Rio de Janeiro. Anais...Rio de Janeiro:IBRACON, pp.1-16.2006.

[27] PRADO,A.G. "Avaliação petrográfica e experimental de agregados graúdos afetados por deformação tectônica, desenvolvimento de minerais de alteração e texturas de exsolução". Dissertação de mestrado. Programa de pós graduação em engenharia mineral. Universidade Federal de Pernambuco. 2008.

[28] GRATTAN-BELLEW P.C., "Concrete prism-CSA A23.2-14A and ASTM C227 mortar bar test". Chapter 3. In:Course manual petrography and alkali-aggregate reactivity. April 20-22,1993, Ottawa, Ontario. pp. 125-160, 1993

[29] SINGH, R.P., UPADHYAY, V.K., DAS, A. ,et al., Weathering potential index for rocks based on density and porosity measurements. Proc. Indian Acad. Sci. (Earth Planet. Sci.), v. 96, n. 3, pp. 239-247, Dec. 1987 ,

[30] SJÖBERG,1999. In: HOLMBERG,R. Explosives \& Blasting Technique. Proceedings of the 1st World Conference on Explosives \& Blasting Technique Munich/Germany/6-8 september. 2000.

\section{ORCID}

Ana Beatriz Azevedo de Medeiros Maria del Pilar Durante Ingunza Antonio Carlos Galindo

Rubens Maribondo do Nascimento https://orcid.org/0000-0003-4175-3255

https://orcid.org/0000-0001-6994-7559

https://orcid.org/0000-0001-9811-2999

https://orcid.org/0000-0001-9094-0044 